DOI: $10.3901 / J M E .2019 .24 .162$

\title{
液压防折弯系统对有轨电车曲线通过 性能影响研究*
}

\author{
黄有培季元进宫岛冷涵任利惠李晶 \\ (同济大学铁道与城市轨道交通研究院 上海 201804)
}

\begin{abstract}
摘要: 依据有轨电车液压防折弯系统工作原理, 基于流量连续性方程和力平衡方程, 并详细考虑缓冲阀工作状态判断依据, 建立有轨电车液压防折弯系统动态模型, 分析液压防折弯系统的动态响应特性。基于液压防折弯系统动态模型, 建立考虑液 压防折弯系统的四模块单车型独立轮对低地板有轨电车协同仿真动力学模型, 研究液压防折弯系统对有轨电车曲线通过性能 的影响。结果表明, 与传统无液压防折弯系统的有轨电车相比, 安装液压防折弯系统可使车辆同一单元内前、后模块车体的 摇头角趋于一致, 同时有效减小车辆最大摇头角及横向偏移量; 防折弯系统控制液压缸作用力不同, 前车靠近导向轮一侧控 制液压缸作用力最大; 液压防折弯系统改善车辆曲线通过性能, 提高整个车辆的安全性。
\end{abstract}

关键词: 有轨电车; 防折弯系统; 动力学; 液压

中图分类号: U270

\section{Influence of Hydraulic Anti-kink System on Curving Performance of Tram}

\author{
HUANG Youpei JI Yuanjin GONG Dao LENG Han REN Lihui LI Jing
}

(Institute of Rail Transit, Tongji University, Shanghai 201804)

\begin{abstract}
According to the action principle of tram hydraulic anti-kink system, based on the flow continuity equation and force balance equation, and considering the judgment basis for the working condition of the buffer valve in detail, a dynamic model of the tram hydraulic anti-kink system is established, and the dynamic response characteristics of hydraulic anti-kink system is analyzed. Based on the dynamic model of hydraulic anti-kink system, the collaborative dynamics simulation model of the tram with 4 modular single carbodies and independently rotating wheels and the hydraulic anti-kink system is established to investigate the influence of the hydraulic anti-kink system on the running performance of tram. The results indicate that: compared with the traditional tram without hydraulic anti-kink system, the installation of hydraulic anti-kink system can make the carbody yaw angle of the front and rear modules in the same unit of the tram tend to be consistent, and effectively reduce the maximum of carbody yaw angle and lateral offset of the tram; the forces applied on anti-kink control cylinders are different and the maximum of the forces is on the control cylinders closed to guide wheel of the front module; hydraulic anti-kink system improves the curving performance and increases safety of the whole tram.
\end{abstract}

Key words: tram; anti-kink system; dynamics; hydraulic

\section{0 前言}

低地板有轨电车作为一种新型的城市公共交通 工具，具有乘降方便、舒适度高、绿色环保、线路 适应能力强等优点, 其最大运量为公交车辆的 $6 \sim 8$ 倍, 造价只有地铁 $1 / 5 \sim 1 / 10$, 在世界各地得到越来 越多的应用 ${ }^{[1-2]}$ 。

\footnotetext{
* “十二五” 国家科技支持计划资助项目(2015BAG19B02)。20180509 收 到初稿, 20190327 收到修改稿
}

低地板有轨电车有多种类型 ${ }^{[3-4]}$, 其中最常见的 是浮动车体型和单车体型。浮动车体是指车体下方 没有转向架支撑, 而是通过铰接装置吊挂在相邻车 体上的车体。浮动车体型有轨电车具有奇数个的车 体模块(3、5、7), 最常见是五模块车体和三个转向 架的编组形式。单车体型有轨电车的特点是每个车 体模块下都仅有一个转向架, 且位于车体模块的中 部。通常两节车体模块编组成一个单元, 每个单元 内的车体模块允许相对转动, 单元与单元之间采用 纵向埽引杆来传递纵向力。单车体型有轨电车通常 采用偶数个数 $(2 、 4 、 6)$ 的编组形式。 
由于一个单元内的两个转向架之间只有一个铰 接点, 单车体型有轨电车通过小曲线的能力明显受 到转向架与车体之间回转约束的限制, 在由直线进 入圆曲线或由圆曲线进入直线的过程中, 电车车体 容易超出限界 ${ }^{[4]}$ 。如图 1 所示, 电车从右向左进入 曲线, $\mathrm{Mc} 2$ 车车体摇头角容易过大, 使得车体最大 横向偏移量(如图 1 中所示 1 4 点位置)过大, 而导 致车辆曲线通过时存在限界超标隐患。

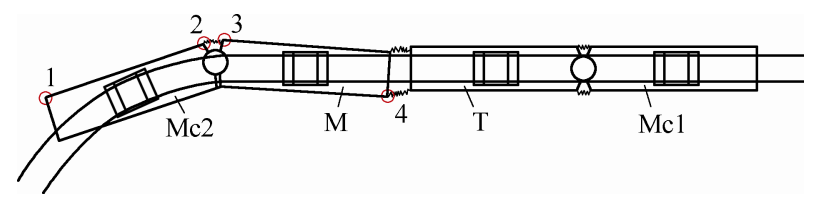

图 1 单车体型有轨电车曲线通过俯视图

为了解决上述问题, 单车体型有轨电车引入了 液压防折弯系统 ${ }^{[5]}$ 。工作原理简图如图 2 所示。其 中, 液压防折弯系统包括横向控制液压缸与相关油 路。车体与转向架之间设置横向控制液压缸, 同一 单元内的两个车体模块间油路 $Z_{1}$ 与 $Z_{2}$ 反对称连 接。通过旁通油路 $Z_{1}$ 和 $Z_{2}$, 使控制液压缸腔体 $V_{1}$ 和 $V_{3}, V_{2}$ 和 $V_{4}$ 分别相连, 并使腔体体积 $V_{1}+V_{3}=V_{2}+V_{4}$ 。当车辆通过图 2 所示曲线时, 一单 元中前车转向架随曲线产生转动, 并与车体产生相 对转角, 控制液压缸活塞压迫至一侧, 前车控制液 压缸腔体 $V_{3}$ 容积减小, 其油液经油路 $Z_{1}$ 流入同单 元后车液压缸腔体 $V_{1}$, 后车控制液压缸在油液压 力作用下将推动活塞使腔体 $V_{2}$ 容积减小, 其油液 又流入至前车控制液压缸腔体 $V_{4}$ 中, 从而使得一 个单元内前、后车体与转向架的转角相等, 即 $\alpha_{1}=\alpha_{2}$ 。

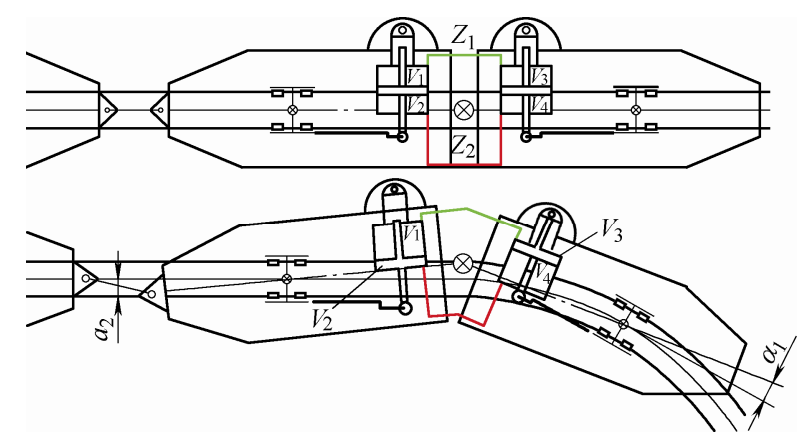

图 2 液压防折弯工作原理简图

有轨电车液压防折弯系统具有防折弯和横向减

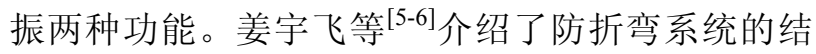
构, 并进行了有轨电车曲线通过试验, 验证了防折 弯系统的作用原理; LI 等 ${ }^{[7-8]}$ 分析了液压防折弯系统 的刚度特性和阻尼特性, 指出液压防折弯系统的刚 度特性和阻尼特性均具有非线性特性; 张徐等 ${ }^{[9-10]}$ 采用了简化的线性阻尼模型和线性弹簧模型模拟防
折弯系统动态特性, 研究了液压防折弯系统对有轨 电车曲线通过性能的影响, 但模型中没有考虑防折 弯系统非线性特性, 无法准确体现液压缸的实际作 用。在既往研究中, 或仅从液压系统的角度研究了 防折弯系统的特性, 没有给出防折弯系统对车辆动 力学性能的影响; 或在动力学分析中将防折弯系统 简化为线性弹簧和阻尼, 无法反映出防折弯系统的 非线性特性。本文基于流量连续性方程和力平衡方 程建立防折弯系统的理论模型, 该模型集刚度和阻 尼特性于一体, 通过联合 Simulink 和 Simpack 软 件建立了协同仿真模型，分析了防折弯系统对有 轨电车曲线通过性能的影响。该研究可为液压防 折弯系统对有轨电车动力学研究提供新思路、新 方法。

\section{1 液压防折弯系统的数学模型}

\section{1 防折弯系统的结构及其布置}

图 3 为单车体型有轨电车的一个单元液压防折 弯系统结构及布置示意图。图中防折弯系统由四个 控制液压缸 3、4、9、10, 两个缓冲阀门组 6、12, 一个旁通节流阀 11 , 一个缓冲液压缸 5 以及旁通油 路 $Z_{1}$ 和 $Z_{2}$ 组成。

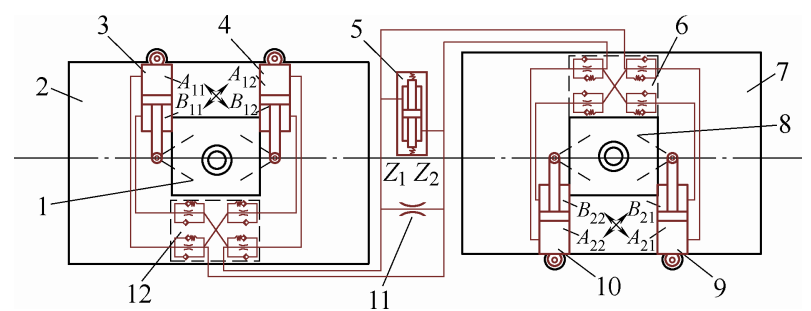

图 3 防折弯系统的结构及布置示意图

1、8. 转向架 $2 、 7$. 前、后车体 $3 、 4 、 9 、 10$. 控制液压缸

5. 缓冲液压缸 $6 、 12$. 缓冲阀门组 11 . 旁通节流阀 $Z_{1} 、 Z_{2}$. 旁通油路

控制液压缸分为两组 $3 、 4$ 和 $9 、 10$, 分别布置 在前、后两个模块车体和转向架之间。控制液压缸 一端通过缸筒与车体连接, 另一端通过活塞与转向 架连接。且两组控制液压缸分别分布在转向架中心 和车体中心两侧, 当车体与转向架横向相对运动时, 活塞杆同向运动, 当车体和转向架相对转动时, 活 塞杆反向运动。

两组控制液压缸分别有四个液压腔 $\left(A_{11} 、 B_{11}\right.$ 、 $A_{12} 、 B_{12}$ 及 $\left.A_{21} 、 B_{22} 、 A_{22} 、 B_{21}\right)$, 斜对角的液压腔通 过两个方向相反的缓冲阀相互连通, 即 $A_{11}$ 和 $B_{12}$ 、 $B_{11}$ 和 $A_{12} 、 A_{21}$ 和 $B_{22} 、 B_{21}$ 和 $A_{22}$ 分别连通, 记为 $A_{1}$ 、 $B_{1}$ 和 $A_{2} 、 B_{2}$ 。当某个模块车体和转向架产生横向相 对运动时, 由于两个缓冲阀的存在, 液压腔 $A_{1} 、 B_{1}$ 
或 $A_{2} 、 B_{2}$ 内油液流动受阻, 使得活塞两侧的油液产 生压差, 油液通过活塞杆和缸筒将作用力传递到相 连的转向架和车体, 阻碍车体和转向架的横向相对 运动。该阻碍横向相对运动的能力即为防折弯系统 的阻尼特性, 方程如下

$$
F=f(v)
$$

式中, $F$ 为阻尼力; $v$ 车体与转向架横向相对速度。 两组液压缸分别通过连接在两个反向缓冲阀之 间的旁通油路 $Z_{1}$ 和 $Z_{2}$ 连通, 即 $A_{1}$ 和 $A_{2}, B_{1}$ 和 $B_{2}$ 分别连通, 使得防折弯系统只含有液压腔 $A 、 B$ 。液 压腔 $A 、 B$ 分布在四个活塞的两侧, 当车体和转向 架相对转动而使活塞两侧的油液产生压差时, 油液 将通过活塞杆和缸筒将作用力传递到相连的转向架 和车体, 抑制或促进车体和转向架之间的相对转动。 将该抑制或促进相对转动的能力定义为防折弯系统 的刚度特性, 特性方程如下 ${ }^{[7]}$

$$
T=K\left(\alpha_{1}-\alpha_{2}\right)
$$

式中, $T$ 为作用于车体和转向架的力矩; $K$ 为防折 弯系统的刚度; $\alpha_{1} 、 \alpha_{2}$ 分别为两个模块中车体和转 向架相对转角, 如图 4 所示。

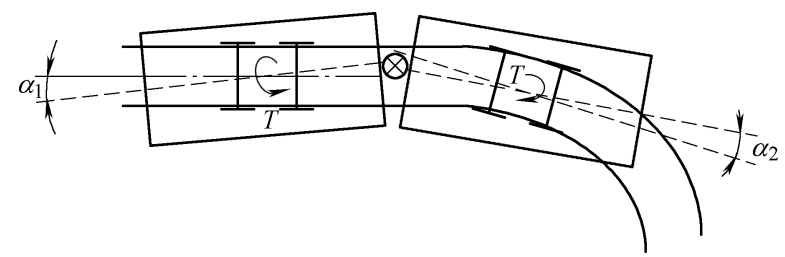

图 4 刚度特性示意图

除此之外, 在旁通油路 $Z_{1}$ 和 $Z_{2}$ 之间安装一个 节流阀和一个受到弹簧约束的液压缸, 称为缓冲缸, 用来调整系统的刚度, 使得防折弯系统的刚度具有 非线性特性，即式(2)中的 $K$ 不为常数。

\section{2 液压防折弯系统的数学模型}

防折弯系统的详细工作原理图如图 5 所示, 图 5 中的变量含义见表 1 。

表 1 图 5 变量的含义

\begin{tabular}{ll}
\hline \multicolumn{1}{c}{ 变量 } & \multicolumn{1}{c}{ 含义 } \\
\hline$q_{1}, q_{2}, q_{3}, q_{4}$ & 流入(出)控制液压缸流量 \\
$q_{\mathrm{r}}$ & 流经旁通节流阀流量 \\
$q_{\mathrm{H}}$ & 流入(出)缓冲缸的流量 \\
$Y_{11}, Y_{12}, Y_{21}, Y_{22}$ & 控制液压缸活塞杆相对于缸筒位移 \\
$p_{\mathrm{A} 11}, p_{\mathrm{A} 12}, p_{\mathrm{A} 21}, p_{\mathrm{A} 22}$ & $\mathrm{~A}$ 腔的油液压强 \\
$p_{\mathrm{B} 11}, p_{\mathrm{B} 12}, p_{\mathrm{B} 21}, p_{\mathrm{B} 22}$ & $\mathrm{~B}$ 腔的油液压强 \\
$p_{1}, p_{2}$ & 旁通油路油液压强 \\
$F_{11}, F_{12}, F_{21}, F_{22}$ & 转向架给与活塞杆作用力 \\
$x$ & 缓冲液压缸活塞杆位移 \\
\hline
\end{tabular}

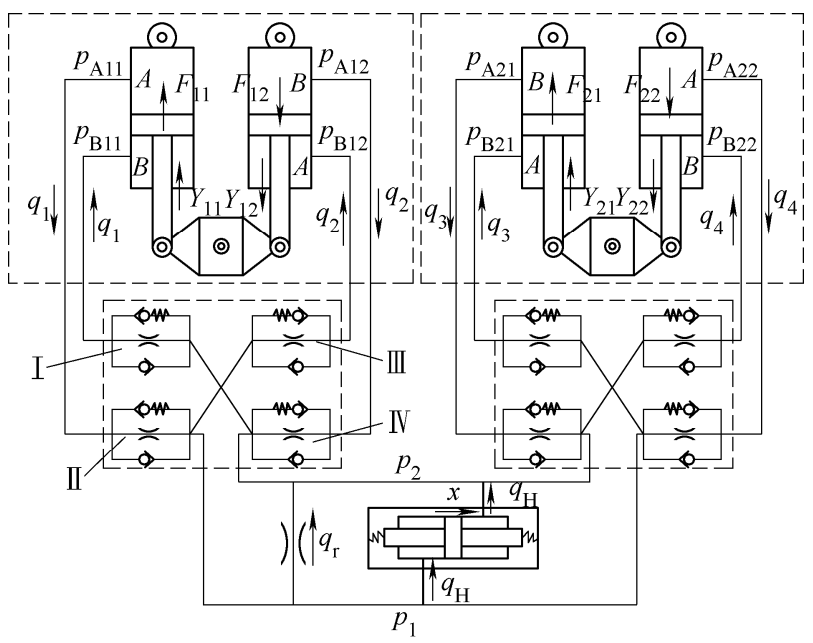

图 5 液压防折弯系统详细原理图 $A 、 B$. 液压腔编号 I、II、III、IV. 缓冲阀编号

\subsection{1 流量连续性方程}

根据图 5 所示的原理图, 将油液视为不可压缩 的理想液体。由流量连续性方程有

$$
q_{1}+q_{2}=q_{3}+q_{4}+q_{\mathrm{r}}+q_{\mathrm{H}}
$$

式中, 控制液压缸处流量 $q=A_{0}(\mathrm{~d} y / \mathrm{d} t), A_{0}$ 为控 制液压缸活塞面积, $Y$ 为控制液压缸活塞杆相对 于缸筒位移; 缓冲液压缸处流量 $q_{\mathrm{H}}=A_{\mathrm{H}}(\mathrm{d} x / \mathrm{d} t)$, $A_{\mathrm{H}}$ 为缓冲液压缸活塞面积; 旁通节流阀处流量 $q_{\mathrm{r}}=C_{\mathrm{d}} A_{\mathrm{d}} \sqrt{\frac{2}{\rho}\left|p_{1}-p_{2}\right|}, C_{\mathrm{d}}$ 为节流口流量系数, $A_{\mathrm{d}}$ 为旁通节流阀节流孔面积。

\subsection{2 力平衡方程}

控制液压缸活塞的力平衡方程

$$
m_{\mathrm{k}} \ddot{Y}=\left(p_{\mathrm{B}}-p_{\mathrm{A}}\right) A_{0}+F-B \dot{Y}
$$

式中, $m_{\mathrm{k}}$ 为控制液压缸活塞质量; $F$ 为转向架给与 活塞杆作用力; $B$ 为液压缸活塞阻尼系数。

同样的, 缓冲液压缸活塞的力平衡方程为

$$
m_{\mathrm{H}} \ddot{x}=\left(p_{1}-p_{2}\right) A_{\mathrm{H}}-2 k x-B \dot{x}
$$

式中, $m_{\mathrm{H}}$ 为缓冲缸活塞质量; $k$ 为缓冲缸弹簧刚度。

\subsection{3 缓冲阀工作状态判断}

图 3 中所示的缓冲阀门组 9、10 分别由四个缓 冲阀组成, 每个缓冲阀的工作原理图如图 6 所示。

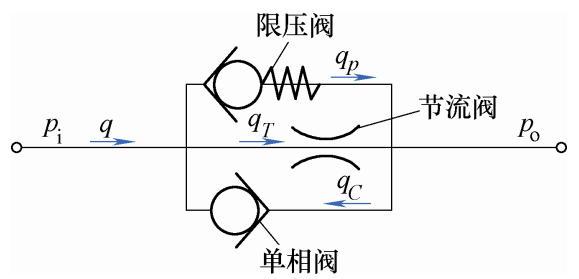

图 6 缓冲阀的工作原理图

缓冲阀由节流阀、向阀和限压阀组成。节流阀 
有增大缓冲力的作用, 而单向阀的作用是使得缓冲 阀门组在如图 6 所示方向油液流动时节流, 反向时 不节流。限压阀的作用是限制缓冲力的大小。当缓 冲阀两端油压超过一定值的时候, 限压阀打开, 一 部分油液便可以经过限压阀流出。

流经节流阀和限压阀的流量分别为 ${ }^{[1-12]}$

$$
\begin{aligned}
& q_{\mathrm{T}}=C_{\mathrm{d}} A_{1} \sqrt{\frac{2}{\rho}\left|p_{\mathrm{i}}-p_{\mathrm{o}}\right|} \\
& q_{\mathrm{p}}=C_{\mathrm{p}}\left(\left|p_{\mathrm{i}}-p_{\mathrm{o}}\right|-\Delta p\right)
\end{aligned}
$$

式中, $p_{\mathrm{i}} 、 p_{\mathrm{o}}$ 为缓冲阀两端的压强; $q_{\mathrm{T}}$ 为流经节流 阀流量; $A_{1}$ 为节流阀节流口面积; $\rho$ 为油液密度; $\Delta p$ 为限压阀开启压强; $q_{\mathrm{p}}$ 为流经限压阀的流量; $C_{\mathrm{p}}$ 为限压阀流量系数。

对于该限压阀而言, 限压阀刚刚开启的瞬间, 流量为

$$
q_{\Delta \mathrm{p}}=q_{\mathrm{T}}=C_{\mathrm{d}} A_{1} \sqrt{\frac{2 \Delta p}{\rho}}
$$

记此时的流量 $q_{\Delta \mathrm{p}}$ 为临界流量, 假设流入缓冲阀 的流量为 $q$, 则通过进入缓冲阀的流量可以判断该缓 冲阀的工作状态: 当 $q<0$ 时, 油液反向流动, 单向 阀起作用; 当 $0<q<q_{\Delta \mathrm{p}}$ 时, 油液正向流动, 且限压 阀未开启, 油液流经节流划; 当 $q_{\Delta \mathrm{p}}<q$ 时, 油液正 向流动, 且限压阀开启, 油液流经节流阀和限压阀。

\subsection{4 系统动态方程}

当前、后车辆分别进入曲线时, 由于前、后车 辆与转向架之间的相对运动, 使得防折弯系统控制 液压缸活塞杆产生位移分别为 $Y_{11} 、 Y_{12} 、 Y_{21} 、 Y_{22}$, 液压缸中的液体发生流动, 流量为活塞面积与活塞 速度的乘积, 即 $q=A_{0}(\mathrm{~d} Y / \mathrm{d} t)$ 。由于缓冲阀的存在, 阀门组的工作状态难以确定, 因此需要对每一个液 压缸的流量进行讨论。

以液压缸 1 为例进行讨论。其余三个液压缸原 理与之相同。

(1) 当控制液压缸的流量 $q_{1}<-q_{\Delta \mathrm{p}}$ 时, 控制液 压缸 $1 A$ 腔油液回流, $B$ 腔外流。此时, 缓冲阀 I 单 向阀关闭, 限压阀和节流阀均开启, 通过节流阀的流 量为 $q_{\mathrm{T}}=q_{\Delta \mathrm{p}}$, 流过限压阀的流量为 $q_{\mathrm{p}}=-\left(q_{1}+q_{\mathrm{T}}\right)$, 缓冲阀两端的压强差满足 $p_{\mathrm{B} 11}-p_{2}=\Delta p+\left(q_{\mathrm{p}} / C_{\mathrm{p}}\right)$, 缓冲阀 II 单向阀打开, 限压阀和节流阀不起作用, 此时, $p_{\mathrm{A} 11}=p_{1}$ 。

结合式(4)求得转向架给与活塞杆的作用力

$$
\begin{gathered}
F_{11}=m_{\mathrm{k}} \ddot{Y}_{11}+B \dot{Y}_{11}- \\
{\left[-\left(p_{1}-p_{2}\right)+\Delta p-\frac{q_{1}+q_{\Delta \mathrm{p}}}{C_{\mathrm{p}}}\right] A_{0}}
\end{gathered}
$$

（2）当流量 $-q_{\Delta \mathrm{p}}<q_{1}<0$ 时, 液压缸 1 的油液流动 情况同情况(1)相同。此时, 缓冲阀 I 单向阀, 限压阀 关闭, 节流阀开启, 流过节流阀的流量为 $q_{\mathrm{T}}=-q_{1}$, 缓 冲阀 I 两端的压强差为 $p_{\mathrm{B} 11}-p_{2}=\left(q_{\mathrm{T}} / C_{\mathrm{d}} A_{1}\right)^{2}(\rho / 2)$, 缓冲阀 II 单向阀开启, 节流阀和限压阀关闭,

$p_{\mathrm{A} 11}=p_{1}$ 。

此时, 转向架给与活塞杆的作用力

$F_{11}=m_{\mathrm{k}} \ddot{Y}_{11}+B \dot{Y}_{11}-\left[-\left(p_{1}-p_{2}\right)+\left(\frac{q_{1}}{C_{\mathrm{d}} A_{1}}\right)^{2} \frac{\rho}{2}\right] A_{0}$

(3) 当流量 $0<q_{1}<q_{\Delta \mathrm{p}}$ 时, 液压缸 $1 A$ 腔油液外流, $B$ 腔回流, 此时缓冲阀 I 单向阀开启, 节流阀和限压阀 关闭。此时有 $p_{\mathrm{B} 11}=p_{2}$; 缓冲阀 II 单向阀、限压阀关 闭, 节流阀开启, 此时通过节流阀的流量为 $q_{\mathrm{T}}=q_{1}$, 缓 冲阀 II 两端的压差为 $p_{\mathrm{A} 11}-p_{1}=\left(q_{\mathrm{T}} / C_{\mathrm{d}} A_{1}\right)^{2}(\rho / 2)$ 。

此时转向架给与活塞杆的作用力为

$F_{11}=m_{\mathrm{k}} \ddot{Y}_{11}+B \dot{Y}_{11}-\left[-\left(p_{1}-p_{2}\right)-\left(\frac{q_{\mathrm{T}}}{C_{\mathrm{d}} A_{1}}\right)^{2} \frac{\rho}{2}\right] A_{0}$

（4）当流量 $q_{\Delta \mathrm{p}}<q_{1}$ 时, 液压缸油液流动与情况 (c)相同, 此时缓冲阀 I 单向阀开启, 节流阀和限压 阀关闭, $p_{\mathrm{B} 11}=p_{2}$; 缓冲阀 II 单向阀关闭, 节流阀 和限压阀均开启, 此时通过节流阀的流量为 $q_{\mathrm{T}}=q_{\Delta \mathrm{p}}$; 通过限压阀的流量为 $q_{\mathrm{p}}=q_{1}-q_{\mathrm{T}}$ 缓冲阀 两端的压差为 $p_{\mathrm{A} 11}-p_{1}=\Delta p+\left(q_{\mathrm{p}} / C_{\mathrm{p}}\right)$ 。

此时, 转向架给与活塞杆的作用力

$$
\begin{gathered}
F_{11}=m_{\mathrm{k}} \ddot{Y}_{11}+B \dot{Y}_{11}- \\
{\left[-\left(p_{1}-p_{2}\right)-\Delta p-\frac{q_{1}-q_{\Delta \mathrm{p}}}{C_{\mathrm{p}}}\right] A_{0}}
\end{gathered}
$$

总结以上工作状态, 控制液压缸 1 的响应方程 如下

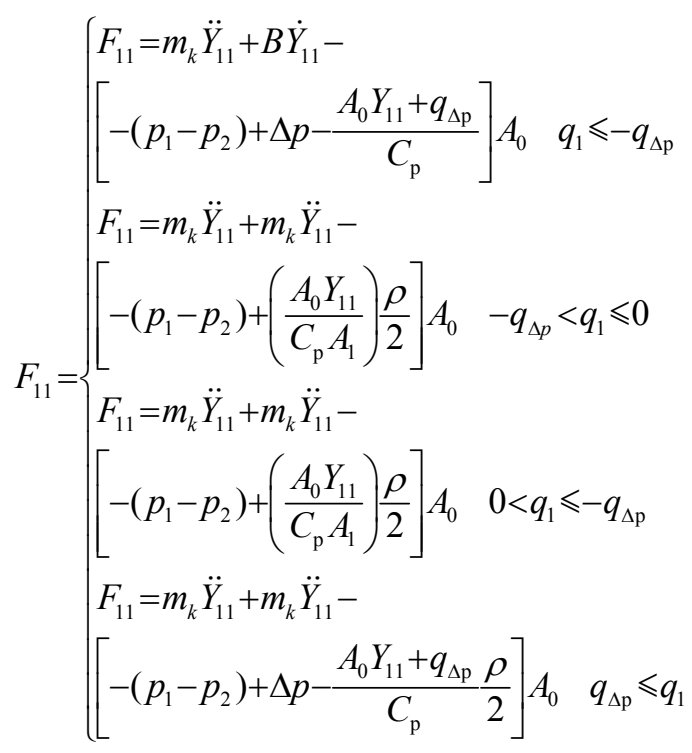


方程式(13)之中包含了变量 $p_{1} 、 p_{2}$, 通过联立 方程式(3)与方程式(5) 可以得到变量 $\left(p_{1}-p_{2}\right)$ 关于 $Y$ 的函数关系。同理可以得到液压缸 $2 、 3 、 4$ 的 相应方程。

\section{2 液压防折弯系统的仿真模型和响应} 特性

\section{1 液压防折弯系统的仿真模型}

基于上述的方程, 使用 Simulink 建立液压防折 弯系统的仿真模型(图 11 右侧)。模型的输入为活塞 杆相对于缸筒位移 $Y_{11} 、 Y_{12} 、 Y_{21} 、 Y_{22}$, 输出为转向 架给与活塞杆作用力 $F_{11} 、 F_{12} 、 F_{21} 、 F_{22}$ 。位移和力 的方向定义同图 5 所示。

\section{2 系统响应特性}

如第 1.1 节所述, 当车体和转向架之间产生横 向相对运动时, 防折弯系统将产生阻尼作用。当前 车车体和转向架之间产生横向的相对运动, 而后车 两者静止时, 防折弯系统受到如图 7a 所示输入。图 $7 \mathrm{a}$ 中, $Y_{11}=-Y_{12}=0.0375 \sin \pi t, Y_{21}=Y_{22}=0$ 。图 $7 \mathrm{~b}$ 为系统在图 $7 \mathrm{a}$ 输入下的响应。由图可知 $F_{11} 、 F_{12}$ 有曲线输出, 且 $F_{11}=-F_{12}, F_{21} 、 F_{22}$ 为 0 。参考图 5 方向可知, 转向架给与活塞杆作用力方向与活塞杆 相对于缸筒位移方向相同, 因而活塞杆给与转向架 的反作用力与活塞杆相对于缸筒位移方向相反, 阻 碍了活塞杆与缸筒的相对运动, 即阻碍了转向架和 车体的相对运动。而由于后车车体和转向架之间无 相对运动, 两者之间无作用力产生。将图 7a 的输入 转化为速度信号绘制力-速度曲线, 如图 8 所示。可 以看到, 该曲线有明显的转折点 $T$, 在转折点 $T$ 以 下, 曲线斜率迅速增大, 在 $T$ 点以上, 曲线斜率保 持不变, 且斜率值较小。
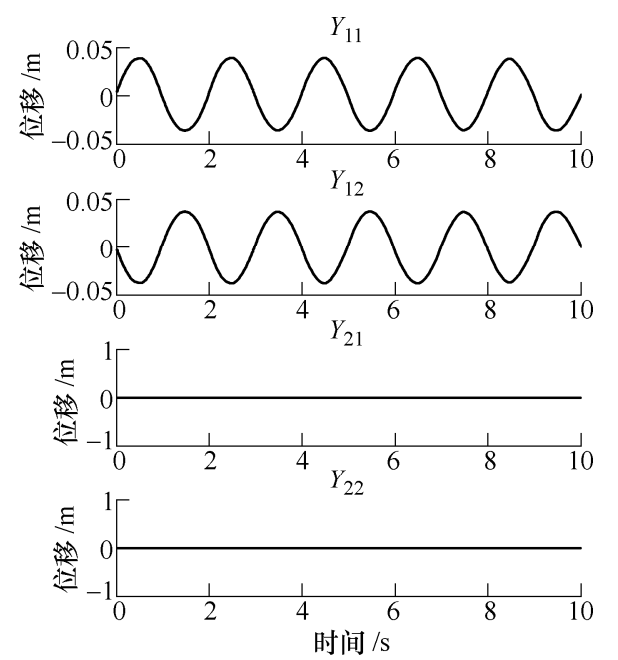

(a) 输入
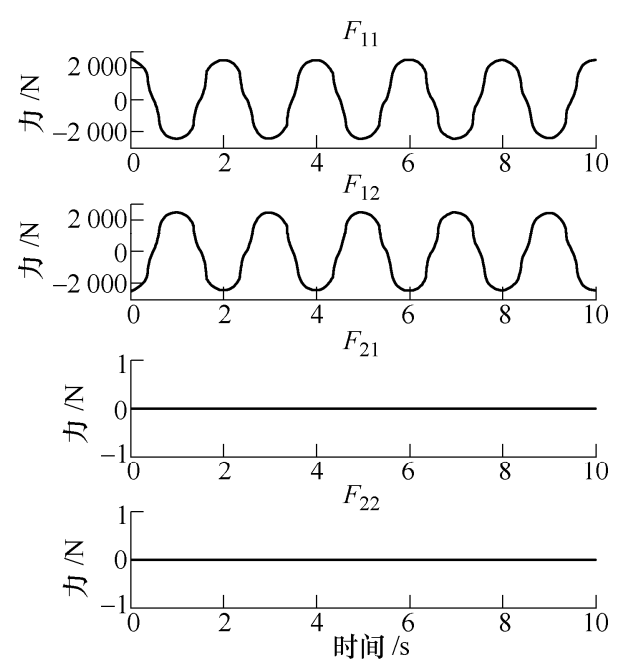

(b) 输出

图 7 横向运动工况下系统响应

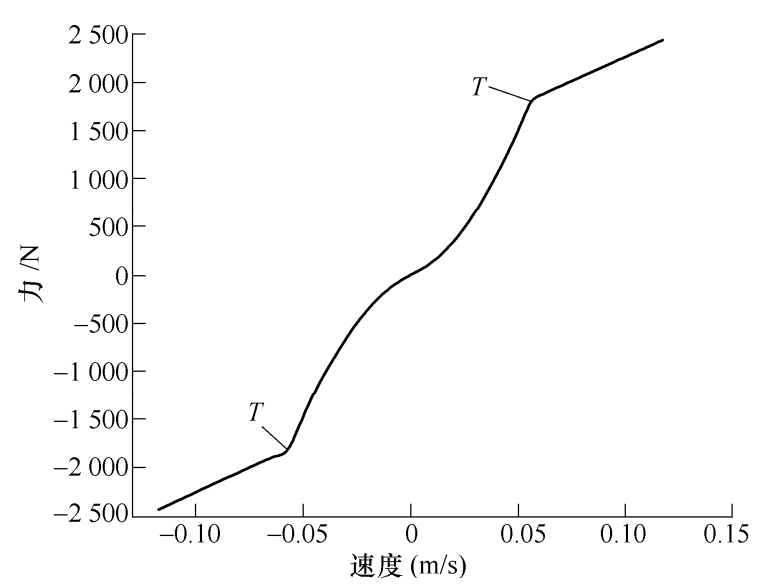

图 8 阻尼特性图 $F-v$

给予系统如图 9a 的斜坡输入, 探究系统的刚 度特性。图 9a 中的输入表示前车控制液压缸 $1 、 2$ 的活塞杆在时刻 $2 \mathrm{~s}$ 以 $0.002 \mathrm{~m} / \mathrm{s}$ 的速度匀速运动 到时刻 $27 \mathrm{~s}$, 之后停止不动直到时刻 $60 \mathrm{~s}$, 后车 控制液压缸活塞杆 3、4 始终保持不动。参考图 5 位移的定义方向, 该输入表示前车车体和转向架 相对匀速转动, 而后车车体和转向架保持静止。

图 $9 \mathrm{~b}$ 中 $F_{11} 、 F_{12} 、 F_{21} 、 F_{22}$ 均有输出, 且 $F_{11}=F_{12}=-F_{21}=-F_{22}$ 。参考图 5 力的定义方向, 前车转向架作用在控制液压缸 1 活塞杆的力与作 用在控制液压缸 2 活塞杆的力方向相反。而根据 图 3, $F_{11} 、 F_{12}$ 和 $F_{21} 、 F_{22}$ 两组力分别位于转向架 和车体中心两侧, 形成以转向架和车体中心为矩 心的力矩。由于 $F_{11} 、 F_{12}$ 符号和 $Y_{11} 、 Y_{12}$ 符号相同, 即转向架给予活塞杆的作用力方向同活塞杆相对 于缸筒的位移方向相同, 因而活塞杆给于转向架 的反作用力方向同活塞杆相对于缸筒的位移方向 
相反, 由此阻碍了前车车体和转向架的相对转动, 而后车的力方向同前车相反，促进了车体和转向 架的相对转动。图 $9 \mathrm{~b}$ 中曲线在活塞杆静止时逐渐 衰减为 0 , 是因为液压腔 $A 、 B$ 存在压差, 使得油 液通过旁通节流阀由 $Z_{2}$ 流向 $Z_{1}$, 减小压差直到液 压腔 $A 、 B$ 压强相同。
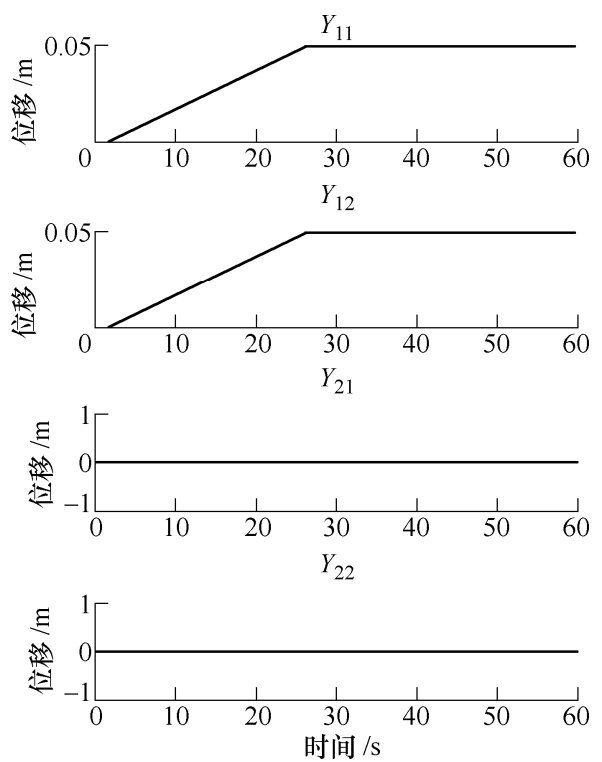

(a) 输入

$F_{11}$
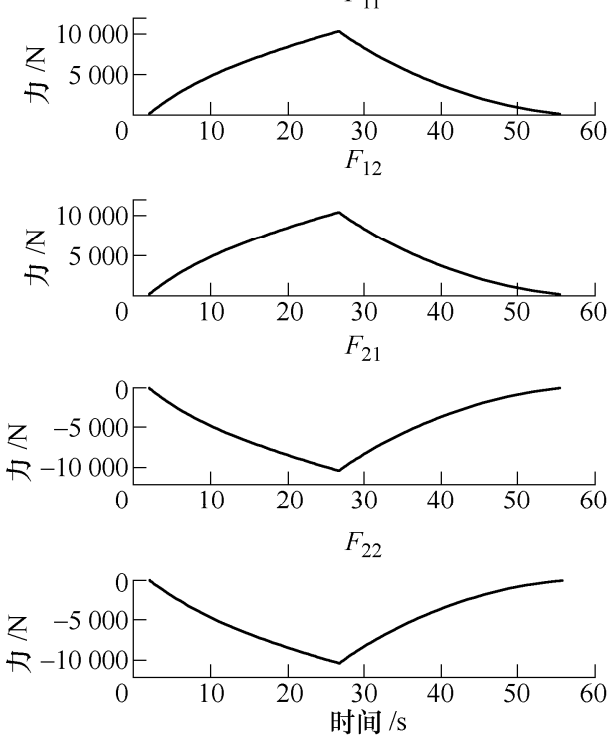

(b) 输出

图 9 转动工况下系统响应

给予系统如图 $10 \mathrm{a}$ 的输入, 得到系统的输出如 图 $10 \mathrm{~b}$ 以探究车体和转向架的相对横向运动及转动 耦合工况下的系统特性。图 10a 的输入为图 $8 \mathrm{a}$ 和图 $9 \mathrm{a}$ 的输入叠加, 图 $10 \mathrm{~b}$ 所示的输出亦是图 $8 \mathrm{~b} 、 9 \mathrm{~b}$ 的输出之叠加。这说明当车辆横向及转动耦合运动 时, 防折弯系统的阻尼和刚度特性互不干扰, 且二 者作用动态叠加。
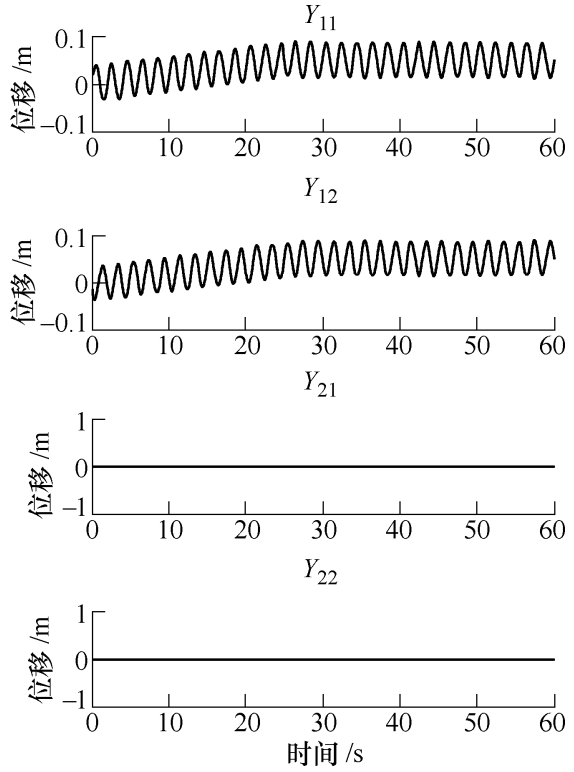

(a) 输入
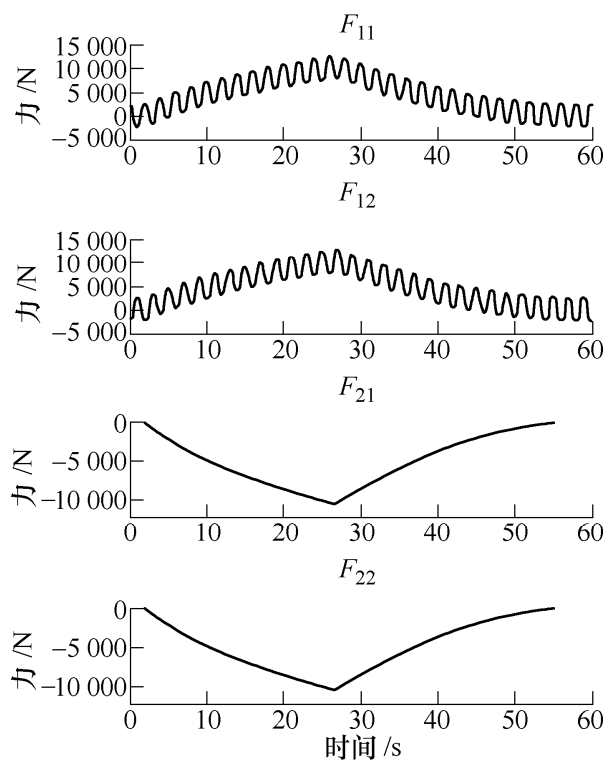

(b) 输出

图 10 横向+转动耦合工况下的系统响应

\section{3 防折弯系统对车辆曲线通过性能影响}

\section{1 动力学仿真模型}

单车体型有轨电车通常由四模块组成, 包括三 节动力模块 $\mathrm{Mc} 1 、 \mathrm{M} 、 \mathrm{Mc} 2$ 及一节拖动模块 $\mathrm{T}$, 如 图 11 所示, 其中 $\mathrm{Mc} 1$ 及 $\mathrm{Mc} 2$ 位于车辆端部。每节 车辆中部设置一个转向架, 其中 $\mathrm{Mc} 2$ 模块与 $\mathrm{M}$ 模 块为一个单元, $\mathrm{Mc} 1$ 模块与 $\mathrm{T}$ 模块为另一单元。 $\mathrm{Mc} 1$ 模块和 $\mathrm{T}$ 模块通过单铰接连接, 单铰接限制了 5 个方向的自由度, 只允许两车之间绕铰接中心在 水平面内转动。 $M$ 模块和 $\mathrm{Mc} 2$ 模块之间与 $\mathrm{Mc} 1$ 模 块和 $\mathrm{T}$ 模块连接相同。 $\mathrm{T}$ 模块和 $\mathrm{M}$ 模块之间采用双 铰连接, 对各自由度没有限制。每个单元安装一套 液压防折弯系统, 如图 3 所示布置。 

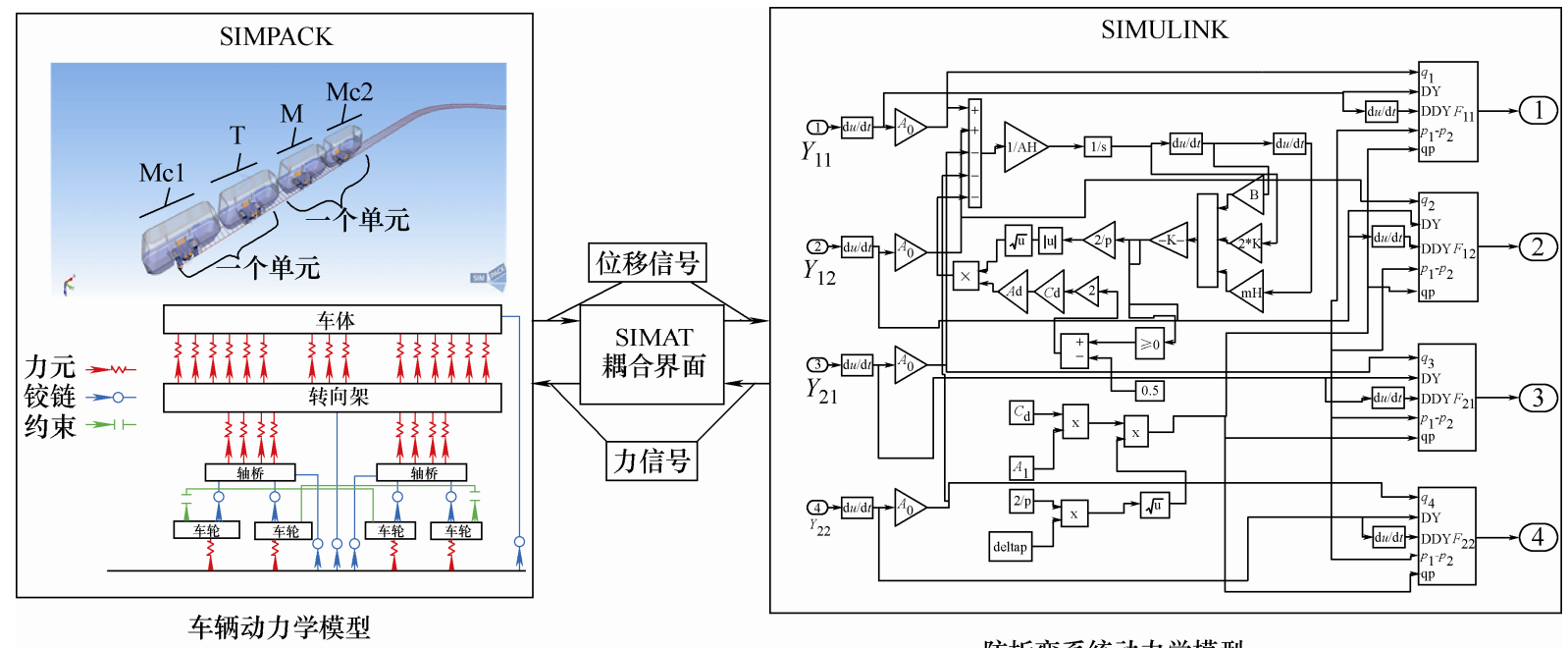

防折弯系统动力学模型

图 11 Simpack+Simulink 联合仿真模型

使用 Simpack 软件建立四模块单车体型有轨电 车的动力学仿真模型, 其中转向架采用纵向耦合的 独立车轮转向架 ${ }^{[13-14]}$, 车辆参数选自文献[10]。将 防折弯系统的 Simulink 仿真模型与四模块单车体型 有轨电车 Simpack 动力学模型进行联合仿真, 仿真模 型如图 11 所示, 图 11 中左侧为有轨电车 Simpack 动 力学模型, 右侧为防折弯系统的 Simulink 仿真模型。

\section{2 动力学仿真结果分析}

根据 GB50090-2006《铁路线路设计规范》以及 CJ/T 417-2012 《低地板有轨电车车辆通用技术条 件》规定要求, 设置车辆曲线通过的仿真工况如下: 初始直线 $50 \mathrm{~m}$, 圆曲线长 $20 \mathrm{~m}$, 半径 $25 \mathrm{~m}$, 末端 直线 $50 \mathrm{~m}$, 曲线超高 $0 \mathrm{~mm}$, 欠超高 $60 \mathrm{~mm}$, 车辆 速度为 $12.6 \mathrm{~km} / \mathrm{h}$ 。

\subsection{1 曲线通过横向偏移量分析}

图 12 给出了车体摇头角的仿真结果。由图 12 可以看出, 无防折弯系统的有轨电车在通过曲线时, 一个单元内两个车体的摇头角曲线明显不同, 模块 $\mathrm{Mc} 1$ 和模块 $\mathrm{M}$ 的车体摇头角明显大于模块 $\mathrm{T}$ 和模 块 $\mathrm{Mc} 2$ 的车体摇头角, 整个车辆中的车体最大摇头 角为 $3.93^{\circ}$, 基本接近车体摇头角设计限值 $\left(4^{\circ}\right)$, 此 情况下, 车体几乎与转向架摇头止档发生接触碰撞, 存在产生轮轨冲击的风险; 而有防折弯系统一个单 元内两个车体模块的摇头角趋于一致, 有较好的重 合度, 模块 $\mathrm{Mc} 1$ 和模块 $\mathrm{M}$ 的车体摇头角稍稍大于 模块 $\mathrm{T}$ 和模块 $\mathrm{Mc} 2$ 的车体偏转角, 整列车辆中的车 体最大摇头角为 $2.92^{\circ}$; 相比无防折弯系统, 有防折 弯系统工况的车体最大摇头角减小了 $1.01^{\circ}$ 。

图 $12 \mathrm{~b}$ 中同一单元内前、后车摇头角趋于一致, 验证了上述基于防折弯系统工作原理建立的动态仿 真模型的正确性。因此, 该动态模型可用于仿真分
析防折弯系统对于有轨电车曲线通过性能的影响。

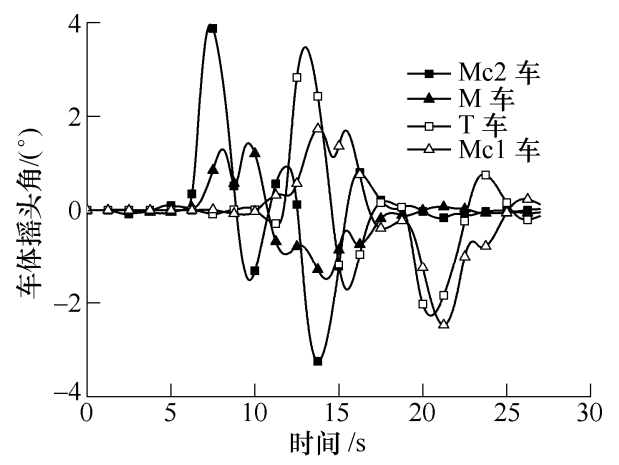

(a) 无防折弯系统

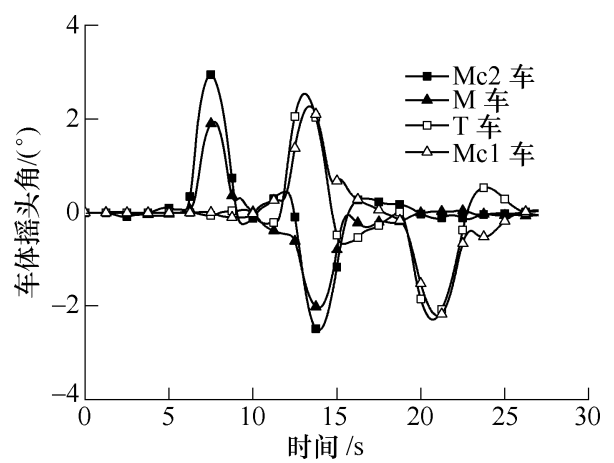

(b) 有防折弯系统

图 12 车体摇头角时间历程

车辆曲线通过时车体最大横向偏移量处位于图 1 所示 $1 、 2 、 3 、 4$ 处。以上四个位置的曲线通过横 向偏移量时域图如图 13 所示, 其中, 初始偏移量为 $1.325 \mathrm{~m}$, 即车体宽度。由于最大横向偏移量值定是 比初始偏移量值更大, 因此, 图 13 省略了 $1.2 \mathrm{~m}$ 以 下的曲线。图 13 中矩形框选中的区域为四个位置横 向偏移量极值区域, 可以看出, 相比无防折弯系统 的工况, 有防折弯系统工下车辆的横向偏移量在位 置 $1 、 2 、 3$ 处较小, 在 4 处较大; 整列车辆的横向 偏移量最大值减少了约 $70 \mathrm{~mm}$ 。 


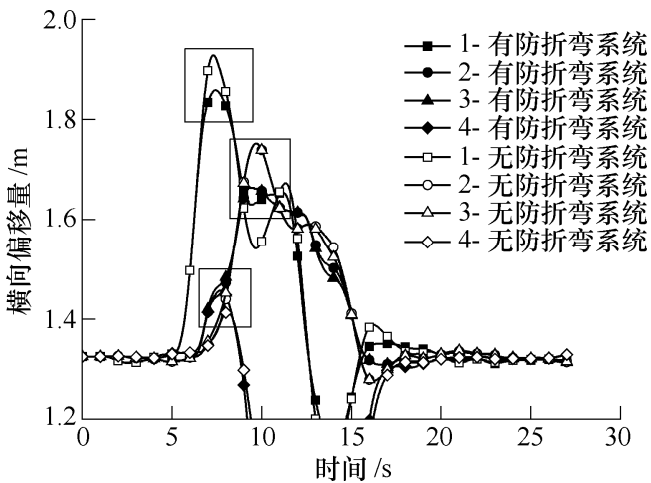

图 13 曲线通过横向偏移量

\subsection{2 曲线通过安全性分析}

图 14 给出了 $\mathrm{Mc} 2$ 模块和 $\mathrm{M}$ 模块导向轮对安全 性指标的时间历程。由图 14 看出, 有、无防折弯系 统时, 车辆通过曲线时轮轴横向力、脱轨系数、磨 耗指数和轮轨横向力的曲线形状基本相同。对于车 辆端部 Mc2 模块的导向轮对, 有防折弯系统的安全 性指标最大值普遍稍小于无防折弯系统的最大值, 而对于 $\mathrm{M}$ 模块的导向轮对, 除了磨耗指数以外, 有 防折弯系统的安全性指标最大值普遍稍大于无防折 弯系统的最大值。这表明, 液压防折弯系统通过增 加车辆中部 $\mathrm{M}$ 模块的轮轨力来减小车辆端部 $\mathrm{Mc} 2$ 模块的轮轨力, 使得整列车辆的轮轨力和脱轨系数 的最大值得以降低。由于轨道车辆曲线通过安全性 评价取决于车辆中安全性指标的最大值 ${ }^{[15]}$ 。因此, 防折弯系统能够提高单车体型有轨电车曲线通过的 安全性。

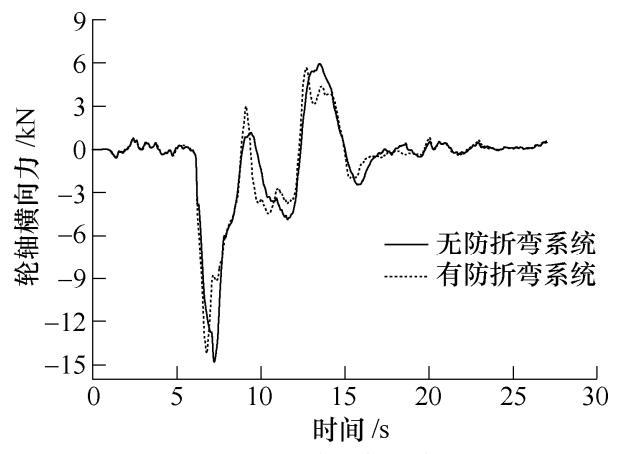

(a) $\mathrm{Mc} 2$ 车轮轴横向力

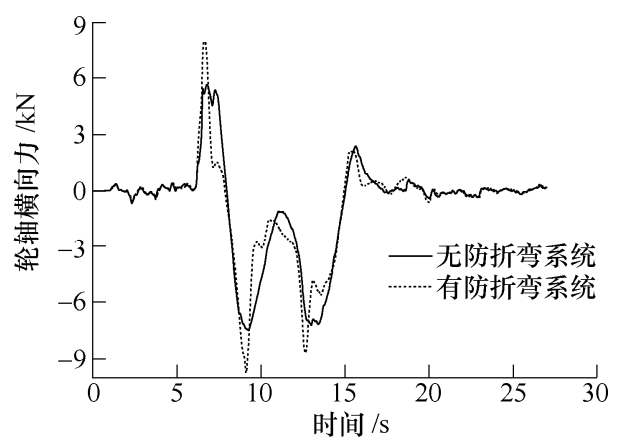

(b) $\mathrm{M}$ 车轮轴横向力

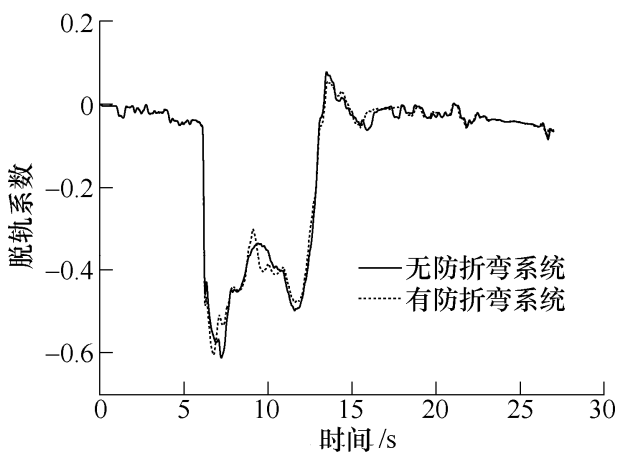

(c) Mc2 车脱轨系数

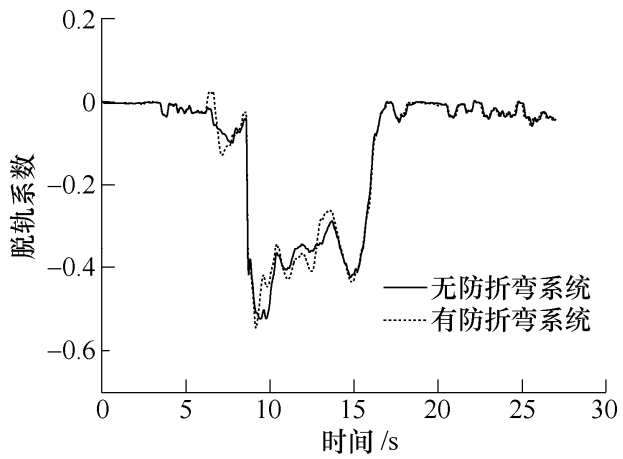

(d) M 车脱轨系数

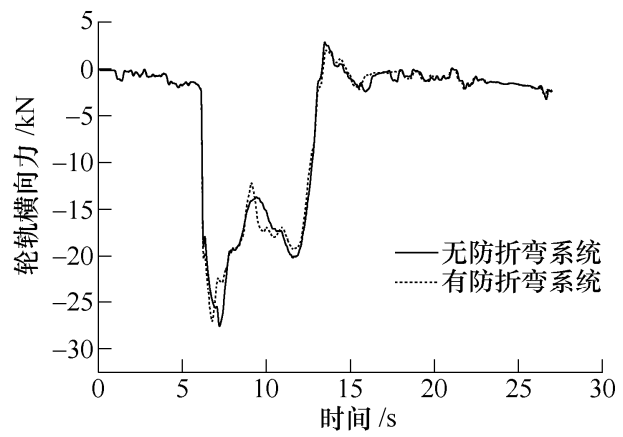

(e) Mc2 车轮轨横向力

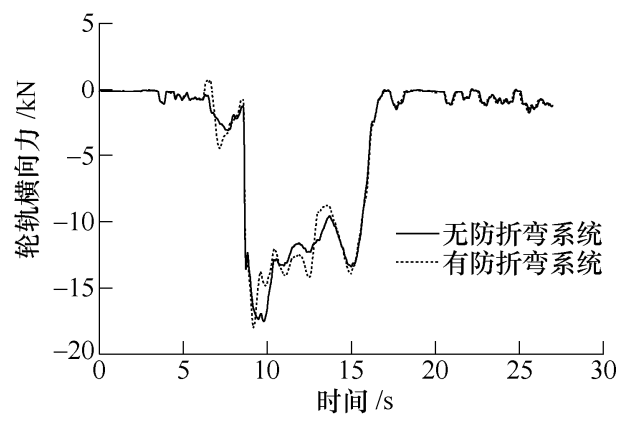

(f) $\mathrm{M}$ 车轮轨横向力

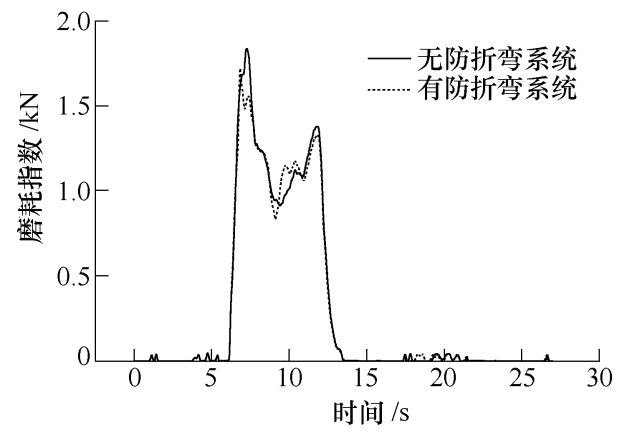

(g) Mc2 车磨耗指数 


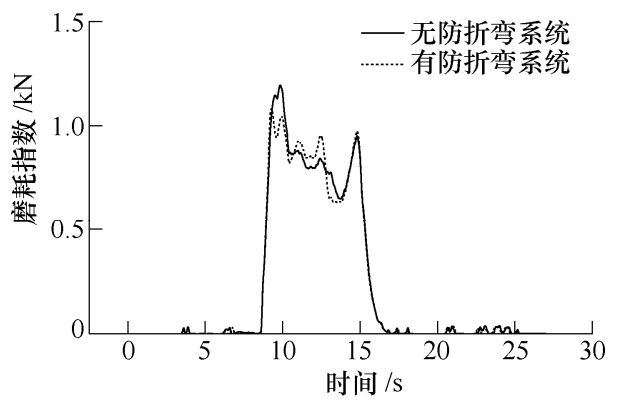

(h) $\mathrm{M}$ 车磨耗指数

图 14 曲线通过安全性时间历程

\subsection{3 防折弯系统作用力分析}

图 15 给出了一个单元内液压防折弯系统中 4 个控制液压缸活塞杆给与转向架的作用力。图 15 中, $F_{11} 、 F_{12}$ 方向与 $F_{21} 、 F_{22}$ 相反, 参考图 5 的方 向, $F_{11} 、 F_{12}$ 阻碍了前车车体和转向架的相对转动, $F_{21} 、 F_{22}$ 促进了后车车体和转向架的相对转动; 四 个液压缸的作用力互不相等, 其中最大值位于曲线 $F_{11}$ 上, 即位于前车靠近导向轮一侧的控制液压缸作 用力最大, 其次为 $F_{12}$ 。

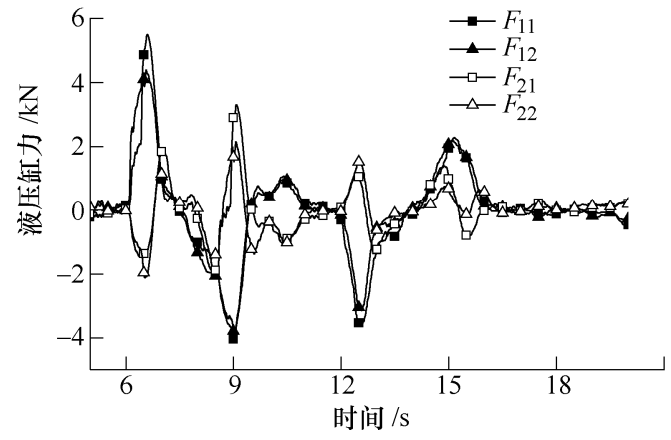

图 15 控制液压缸的作用力

\section{4 结论}

(1) 依据有轨电车液压防折弯系统的工作原 理, 基于流量连续性方程和力平衡方程建立了防折 弯系统的理论模型, 考虑了缓冲阀判断依据, 据此 建立了抗折弯系统动态理论模型, 并分析了该理论 模型的动态响应特性。研究表明, 该模型具有阻尼 和刚度特性, 二者作用动态叠加。

(2) 建立了采用纵向耦合独立轮对的单车型低 地板有轨电车动力学和液压防折弯系统的协同仿真 模型, 研究了液压防折弯系统对有轨电车曲线通过 性能的影响。研究表明, 相比无防折弯系统, 增加 液压防折弯系统可使同一单元的两节车摇头角趋于 一致, 同时有效减小车辆最大摇头角及最大横向偏 移量; 通过增大同一单元后车模块安全性指标, 减 小前车模块安全性指标, 使得整列车辆安全性指标
减小，改善曲线通过安全性。

(3) 防折弯系统在有轨电车曲线通过过程中, 四个控制液压缸给与车体和转向架的作用力不同, 前车靠近导向轮一侧的控制液压缸作用力最大。

\section{参 考 文 献}

[1] HONDIUS H. The development of low-flow trams[J] Journal of Advanced Transportation, 1993, 27(1): 79-102.

[2] 陆云. 现代有轨电车工程 $[M]$. 成都: 西南交通大学出 版社，2015

LU Yun. Modern tram engineering [M]. Chengdu : Southwest Jiaotong University Press, 2015.

[3] 李明. 城市轻轨车辆选型及动力学性能研究[D]. 成都: 西南交通大学, 2004.

LI Ming. Lectotype of light rail vehicles and reasearch on dynamic performance[D]. Chengdu: Southwest Jiaotong University, 2004.

[4] 王欢. $100 \%$ 低地板轻轨车辆结构型式与导向机理研究 [D]. 成都: 西南交通大学, 2008

WANG Huan. Research of structure style and steering principle of $100 \%$ low floor light rail vehicle[D]. Chengdu: Southwest Jiaotong University, 2008.

[5] 姜宇飞, 谭明敏, 王力军. 储能式低地板有轨电车防折 弯系统分析 [J]. 技术与市场，2015(11)：26-27.

JIANG Yufei, TAN Mingmin, WANG Lijun. Anti-kink system of energy storage modern tram[J]. Technology and Market, 2015(11): 26-27.

[6] 黄江伟, 雷新红, 张登科, 等. 储能式现代有轨电车防 折弯系统试验研究 [J]. 技术与市场, 2015(5): 26-27. HUANG Jiangwei, LEI Xinhong, ZHANG Dengke, et al. Experimental study on anti-king system of energy storage modern tram $[\mathrm{J}]$. Technology and Market, 2015(5): 26-27.

[7] LI Jing, ZHU Xianliang, ROBINEAU C. Non-linear stiffness analyzing of a hydraulic assisted turning system in low-floor trams[C]// International Conference on Fluid Power \& Mechatronics. August 5, 2015, Harbin, Heilongjiang, China. Beijing China: FPTCI, CMES, 2015: 518-524.

[8] 李晶, 朱先亮, 任利惠. 低地板轻轨车液压防折弯系统 阻尼特性分析 [J]. 同济大学学报, 2017, 36(7): 1044-1049.

LI Jing, ZHU Xianliang, REN Lihui. Analysis of damping characteristics of hydraulic anti-kink system in low-floor trams[J]. Journal of Tongji University, 2017, 36(7) : 1044-1049.

[9] 张徐. 四模块 $100 \%$ 低地板有轨电车小曲线通过性能研 究[D]. 成都: 西南交通大学, 2016. 
ZHANG Xu. Study on small curve performance of four modules $100 \%$ low-floor trams[J]. Chegndu: Southwest Jiaotong University, 2016.

[10] 吕凤梅, 赵建秋, 间晓明, 等. $100 \%$ 低地板现代城市有 轨电车限界和小曲线通过能力分析 $[\mathrm{J}]$. 铁道车辆, 2013, 51(9): 5-7.

LÜ Fengmei, ZHAO Jianqiu, YAN Xiaoming, et al. Gauge for $100 \%$ low floor modern urban tram and analysis of the small curve negotiation capability[J]. Rolling Stock, 2013, 51(9): 5-7.

[11] DUYM S, STIENS R, REYBROUCK K. Evaluation of shock absorber models [J]. Vehicle System Dynamics, 1997, 27(2): 109127.

[12] 吕振华, 李世民. 筒式液阻减振器动态特性模拟分析技 术的发展[J]. 清华大学学报, 2002, 42(11): 1532-1536. LÜ Zhenhua, LI Shimin. Simulation techniques for nonlinear dynamic characteristics of telescopic hydraulic dampers[J]. Journal of Tsinghua University, 2002, 42(11): 1532-1536.

[13] 王欢, 田合强, 戴焕云. 独立车轮动力转向架纵向耦合 导向机理[J]. 中国铁道科学，2010，31(4): 63-67.
WANG Huan, TIAN Heqiang, DAI Huanyun. The Principle of the longitudinal coupled creep steering for the independently wheel power bogie[J]. China Railway Science, 2010, 31(4): 63-67.

[14] 许明春, 曾京. 纵向耦合独立旋转车轮转向架导向机理 [J]. 交通运输工程学报, 2011，11(1): 43-50.

XU Mingchun, ZENG Jing. Guiding mechanism of longitudinal coupling bogie with independently rotating wheels[J]. China Railway Science, 2011, 11(1): 43-50.

[15] 中华人民共和国国家标准局, 铁道部标准计量研究所. GB/T 5599-1985 铁道车辆动力学性能评定和试验鉴定 规范[S]. 北京: 中国标准出版社, 1985.

National Bureau of Standards, Institute of Standard Metrology. GB/T 5599-1985 Railway vehicles-S pecification for evaluation the dynamic performance and accreditation test[S]. Beijing: China Standard Press, 1985.

作者简介: 黄有培, 男, 1992 年出生, 博士研究生。主要研究方向为轨 道车辆系统动力学。

E-mail: huangyoupei2015@163.com

任利惠(通信作者), 男, 1970 年出生, 博士, 教授, 博士研究生导师。

主要研究方向为轨道车辆系统动力学, 车辆测试技术。

E-mail: renlihui@tongji.edu.cn

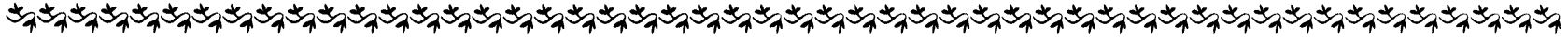

\section{(上接第 161 页)}

风机噪声测量方法 $[S]$. 北京: 中国标准出版社, 2008 . General Administration of Quality Supervision, Inspection and Quarantine of the People's Republic of China, Standardization Administration of the People's Republic of China. GB/T 2888-2008 Methods of noise measurement for fans blowers compressors and Roots blowers[S]. Beijing: Standards Press of China, 2007.

[13] 康强, 左曙光, 韩惠君, 等. 燃料电池车用旋浴风机气 动噪声试验及仿真研究 [J]. 汽车工程, 2014, 36(2): 236-242.

KANG Qiang, ZUO Shuguang, HAN Huijun, et al. Experimental and simulation studies on the aerodynamic noise of regenerative flow compressor for fuel cell vehicle[J]. Automotive Engineering, 2014, 36(2): 236-242.

[14] 张强. 气动声学基础[M]. 北京: 国防工业出版社, 2012. ZHANG Qing. Aero-acoustic foundation[M]. Beijing: National Defense Industrial Press, 2012.

[15] 陈庆光, 房菲, 张永超, 等. 对旋风机级间流场浴量和 噪声分布规律的分析 [J]. 噪声与振动控制, 2012, 2(1): 89-93.

CHEN Qingguang, FANG Fei, ZHANG Yongchao, et al.
Vortex and noise bharacteristics analysis of the flow-field between the two impellers of a contra-rotating axial fan[J]. Noise and Vibration Control，2012， 2(1): 89-93.

[16] LIGHTHILL M J. On sound generated aerodynamically. General theory[J]. Proceedings of the Royal Society of London Series A: Mathematical and Physical Sciences, 1952, 211(1107): 564-587.

[17] 智乃刚, 屈德荫, 蔡京京, 等. 离心通风机的 $\mathrm{A}$ 声级和 倍频带声压级的预算方法[J]. 应用声学，1983，2(4): 36-37.

ZHI Naigang, QU Deyin, CAI Jingjing, et al. Budget method for a sound level and octave sound pressure level of centrifugal fan[J]. Journal of Applied Acoustics, 1983, 2(4): $36-37$.

[18] 星谷胜. 随机振动分析[M]. 北京: 地震出版社, 1977. XING Gusheng. Random vibration analysis[M]. Beijing: Seismological Press, 1977.

作者简介: 刘健炜, 男, 1992 年出生。主要研究方向为轨道交通振动噪 声控制。

E-mail: 542242399@qq.com

圣小珍(通信作者), 男, 1962 年出生, 教授, 博士研究生导师。主要研 究方向为振动噪声理论。

E-mail: shengxiaozhen@hotmail.com 